\title{
Kinematic analysis of tandem gait on a sine wave walkway
}

\author{
Shingo KaWAKami ${ }^{1,2)^{*}}$, Hiroyuki Fujisawa, PRT, PhD ${ }^{1,2)}$, Yoshiyuki Tomizawa, RPT ${ }^{1,3)}$, \\ KenICHI MuraKami, PRT, MS ${ }^{1)}$
}

1) Graduate School of Health and Environment Science, Tohoku Bunka Gakuen University: 6-45-1

Kunimi, Aoba-ku, Sendai-shi, Miyagi 981-8551, Japan

2) Department of Rehabilitation, Faculty of Medical Science and Welfare, Tohoku Bunka Gakuen University, Japan

3) Department of Rehabilitation, Social Medical Corporation Syodokai Minamitohoku General Hospital, Japan

\begin{abstract}
Purpose] The purpose of this study was to ascertain the kinematic characteristics on a horizontal plane, including knee joint rotation, when walking with a tandem gait on a sine wave walkway. [Subjects and Methods] Eighteen healthy adults were enrolled as subjects in this study. They walked with a tandem gait on a sine wave walkway. A three-dimensional motion analysis system was used to record data and calculate the trunk, hip joint, and knee joint rotation angles. [Results] The rotation angle ranges for the trunk, hip joint, and knee joint were $23.3^{\circ}$, $53.3^{\circ}$, and $47.3^{\circ}$, respectively. The trunk generally rotated towards the direction of movement, and when turning left using the left leg as the pivot, the hip joint was internally rotated and the knee joint was externally rotated. In contrast, when making a directional change to the right using the left leg as the pivot, the hip joint was externally rotated and the knee joint was internally rotated. [Conclusion] Through tandem gait analysis on a sine wave walkway, knee joint rotation was found to be important in changes of direction.

Key words: Sine wave walkway, Tandem gait, Knee joint rotation
\end{abstract}

(This article was submitted Feb. 24, 2016, and was accepted May 23, 2016)

\section{INTRODUCTION}

Knee joint rotation is probably important for smooth changes of direction while walking. Although no established method exists to evaluate movement in direction change, the gait ability test of the Meiji Yasuda Life Foundation of Health and Welfare is often used ${ }^{1)}$. In this test, a 10.0-m walkway is set up with two points indicating where to change direction on the left and right, at intervals of $2.0 \mathrm{~m}, 0.5 \mathrm{~m}$ from the center line. At the start signal, subjects walk around the outside of each point indicating to change direction as quickly as possible, and measure the time taken to reach the goal. It is a performance test for which measurements are taken twice and the lower of the two values is adopted. Therefore, it is excellent for comparison with a reference value. Some reports have described this method in evaluating the physical fitness of elderly people ${ }^{2,3)}$ and its association with myofunction ${ }^{4}, 5$. However, the kinematics, specifically of knee joint rotation, during direction change have not been examined.

Studies have clarified that center of pressure (COP) control from active knee joint rotation occurs when maintaining a one-legged stance, which is considered a difficult task in the field of physical therapy ${ }^{6}$. The frequency in the COP control included high contents of $2 \mathrm{~Hz}^{7}$ ). Therefore, the possibility of using a one-legged stance task in the evaluation of knee joint rotation function and its application in exercise therapy with a goal of improving function was suggested.

Examining the association between the ability to maintain a one-legged stance and the ability to change directions when

*Corresponding author. Shingo Kawakami (E-mail: kawakami@rehab.tbgu.ac.jp)

(C2016 The Society of Physical Therapy Science. Published by IPEC Inc.

This is an open-access article distributed under the terms of the Creative Commons Attribution Non-Commercial No Derivatives (by-nc-nd) License $<$ http://creativecommons.org/licenses/by-nc-nd/4.0/>. 


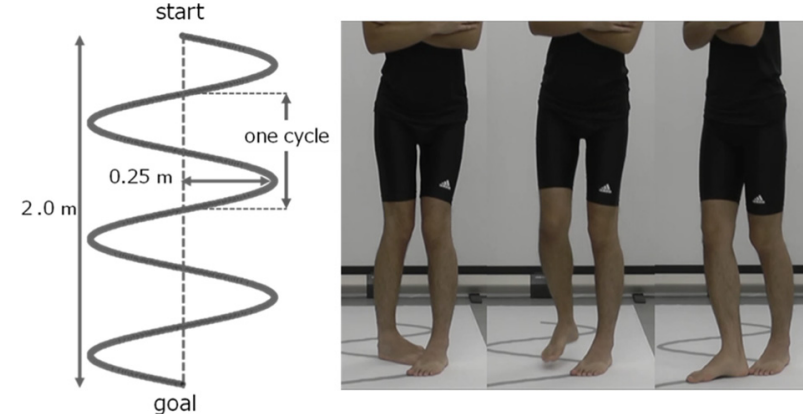

Fig. 1. Tandem gait on a sine wave walkway

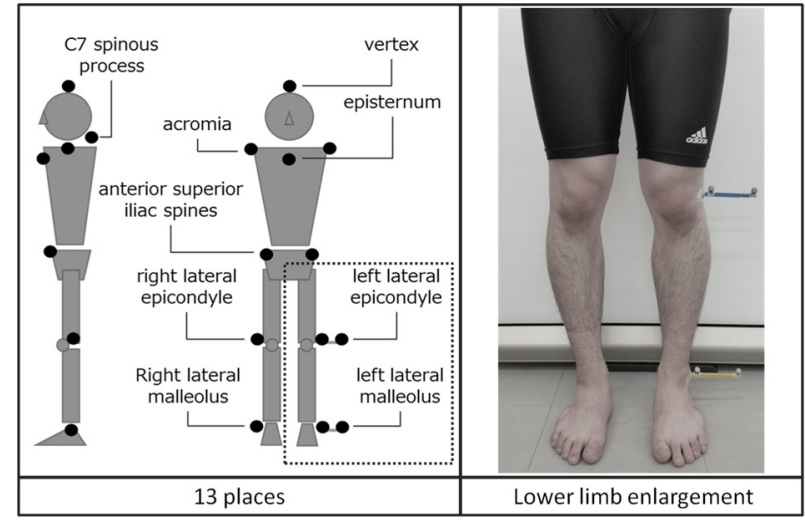

Fig. 2. The 13 locations of the markers

walking is also important in terms of physical therapy. However, first it is necessary to ascertain the extent to which knee joint rotation is used during direction changes when walking. However, the methods used in previous studies analyzing direction change were insufficient in terms of dealing with step width. Therefore, problems arose with the quantitative measurement of knee joint rotation ${ }^{8,9}$. For that reason, we specifically examined the tandem gait using a sine wave walkway.

The purpose of this study was to ascertain the kinematic characteristics on a horizontal plane, including knee joint rotation, when walking with a tandem gait on a sine wave walkway.

\section{SUBJECTS AND METHODS}

Eighteen healthy adults (gender; 12 male, 6 female, age; $21.9 \pm 2.7$ years; height, $167.7 \pm 6.8 \mathrm{~cm}$; weight, $61.6 \pm 8.7 \mathrm{~kg}$ ) were enrolled in the study. The pivot leg was defined as the leg opposite to that used when kicking a ball. It was the left leg for all subjects. Additionally, we confirmed in advance that subjects did not have a current or history of CNS disease that limits walking, orthopedic disease, or visual disturbance or vestibular function disorder that affects balance. We obtained signed consent after explaining the study procedure in detail to all participants.

The movement task involved subjects walking with a tandem gait on a sine wave walkway. The adopted walkway was $2.0 \mathrm{~m}$ long, with an amplitude of $0.25 \mathrm{~m}$, and the sine wave had three cycles. Additionally, the width of the line indicating the walkway was $3.6 \mathrm{~cm}$ (Fig. 1). A six-camera, three-dimensional motion analysis system (Locus 3D MA-5000; Anima Corp.) was used to record movement. The sampling frequency was $150 \mathrm{~Hz}$. First, we affixed markers on the following 13 locations: vertex, C7 spinous process, episternum, both acromia, both anterior superior iliac spines, two places on the left lateral epicondyle, right lateral epicondyle, two places on the left lateral malleolus, and the right malleolus medialis. We standardized the markers that we affixed to the left lateral epicondyle and the left lateral malleolus at positions of $1.5 \mathrm{~cm}$ and $10.0 \mathrm{~cm}$ from each bone index (Fig. 2).

The start position was a standing position on the starting line with arms crossed over the chest. At the sound of a signal, subjects started to walk with a tandem gait. The task ended when both feet stepped over the goal line. In performing this task, we set up three discontinuation criteria: if the folded arms became unfolded; if a foot deviated from the walkway; and if, when in a double-leg support period, a part of the ankle of one foot and the tip of the other foot were not touching the ground. When any of those occurred, we measured the gait again.

We calculated trunk, hip joint, and knee joint rotation angles from one cycle of coordinate data from the $2.0 \mathrm{~m}$ walkway. The following calculation method to obtain the rotation angles was used: the trunk rotation angle is the angle formed by the intersection of the perpendicular line connecting $\mathrm{C} 7$ and the episternum and the line connecting both ASISs. The hip joint rotation angle is the angle formed by the intersection of the line connecting both ASISs and the line that connects the two markers on the left lateral epicondyle of the femur. The knee joint rotation angle is the angle formed by the intersection of the line that connects the two markers on the left lateral epicondyle of the femur and the line that connects the two markers on the left lateral malleolus. In addition, regarding the knee joint rotation angle, the position of the thigh and calf were defined as $0^{\circ}$ in the upright position. For data processing, we used an original program written in MATLAB ${ }^{\circledR}$ (The MathWorks Inc.).

\section{RESULTS}

The angle change in external and internal rotation and angle range are shown in Table 1 . The trunk, hip joint, and knee joint rotation angles were $23.3^{\circ}, 53.3^{\circ}$, and $47.3^{\circ}$, respectively. Figure 3 shows the relationship between each segment angle of the representative example concerned. Foot length differed between subjects. It showed a significantly greater value in 
Table 1. Segment angle

\begin{tabular}{lccc}
\hline & $\begin{array}{c}\text { Angle change } \\
\text { to external } \\
\text { rotation }\left({ }^{\circ}\right)\end{array}$ & $\begin{array}{c}\text { to internal } \\
\text { rotation }\left({ }^{\circ}\right)\end{array}$ & Angle range $\left(^{\circ}\right)$ \\
\hline Trunk rotation & $13.0 \pm 6.4$ & $-10.3 \pm 7.2$ & $23.3(13.0-10.3)$ \\
Hip rotation & $15.6 \pm 10.4$ & $-37.7 \pm 9.4$ & $53.3(15.6-37.7)$ \\
Knee rotation & $18.4 \pm 9.4$ & $-28.9 \pm 12.0$ & $47.3(18.4-28.9)$ \\
\hline
\end{tabular}

Trunk rotation: angle change to right rotation $(+)$ angle change to left rotation $(-)$
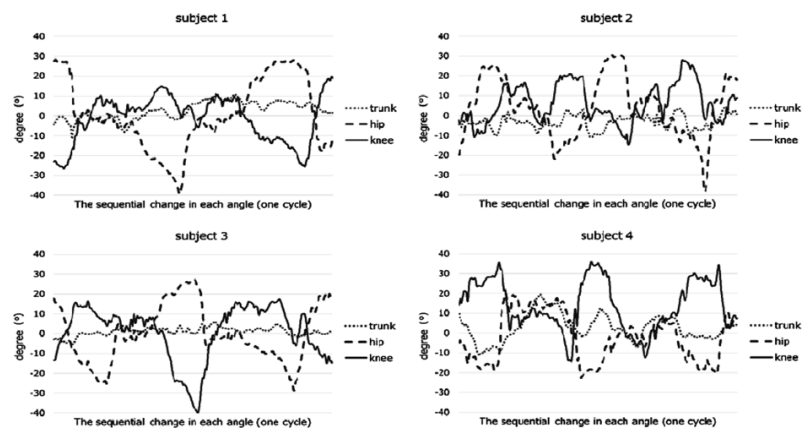

Fig. 3. The relationship between each segment angle of the representative example

Trunk rotation: angle change to right rotation $(+)$ angle change to left rotation (-)

Hip rotation: angle change to external rotation $(+)$ angle change to internal rotation $(-)$

Knee rotation: angle change to external rotation $(+)$ angle change to internal rotation $(-)$

male. The sequential change in each angle varied among subjects, but we were able to confirm the relationship between changes was common to all subjects. The trunk generally rotated towards the direction of movement. When turning left using the left leg as the pivot, the hip joint was internally rotated and the knee joint was externally rotated. In contrast, when making a directional change to the right using the left leg as the pivot, the hip joint was externally rotated and the knee joint was internally rotated. Furthermore, the hip joint and knee joint rotation angles showed multiple peaks and overlapped with the movement at the time of direction change.

\section{DISCUSSION}

Rotation of the knee joint while walking is important for smooth changes of direction. However, the kinematics, especially of knee joint rotation, during direction change have not been examined sufficiently. Therefore, we specifically examined tandem gait in a sine wave walkway and chose to determine the kinematic characteristics on a horizontal plane, including knee joint rotation.

The sine wave walkway was designed based on pre-experimental data. Because the gait was linear during the first and second cycles of the sine wave, we used the one cycle of the sine wave for the calculations of rotation angles. In addition, no difference was found in terms of speed or the number of deviations in the tandem gait with changes in the width of the walkway. Therefore, considering versatility, we adopted a width of $3.6 \mathrm{~cm}$, as marked using commercially available adhesive tape.

As a result of data analysis, each angle range and their relation to the first cycle became evident. Each angle range in the first cycle was as follows: the range of the trunk rotation angle was $23.3^{\circ}$, that of the hip joint rotation angle was $53.3^{\circ}$, and that of the knee joint rotation angle was $47.3^{\circ}$. Furthermore, each angle showed common relations in all subjects. The reference range of motion that the Japanese Orthopedics Association and the Japanese Association of Rehabilitation Medicine established was $90^{\circ}$ rotation for the hip joint and $80^{\circ}$ for the trunk ${ }^{10)}$. The calculated movements were all within the reference range. The reference range of motion is not specified for the rotation of the knee joint. Furthermore, few previous studies have measured the movement of the knee joint three-dimensionally. At present, there is no agreed upon method of measurement. In particularly, the researchers of preliminary studies state that the definition of the position of $0^{\circ}$ is one factor that makes unification of measurement methods difficult. However, approximate angle ranges have been reported, although no consensus has been obtained. Ross measured the passive rotation angles of the knee joint at different angles of flexion in men. He reported that when the knee joint was flexed at $45^{\circ}$, the rotation range was $41^{\circ}$, at $90^{\circ}$, the range was $37^{\circ}$, and at $135^{\circ}$, the range was $39^{\circ 11}$. Ouellet et al. measured the passive rotation angle in the sitting position, and reported that it was $40^{\circ}$ on the right and $43^{\circ}$ on the left ${ }^{12}$. Mossberg et al. measured the active rotation angle in women. They reported that it was $34.9^{\circ}\left(18-55^{\circ}\right)$ when the knee joint was flexed at $70^{\circ}, 40.4^{\circ}\left(20-67^{\circ}\right)$ at $90^{\circ}$, and $44.1^{\circ}\left(24-67^{\circ}\right)$ at $\left.110^{\circ} 13\right)$. Because the knee joint rotation angle ranges in the present study were similar to the values shown in preliminary research, we believe the calculated values to be reasonable. Considering the results described above, it became evident that in terms of knee joint rotation, it requires a wider range of movement compared with the reference range of motion, unlike the trunk or hip joint. Additionally, it is extremely interesting that a definite relation was found for each intersegmental movement. For example, in changing direction to the left with the left leg as the axis, the trunk and the knee joint showed rotation to the left and external 
rotation, which was the direction of movement. However, the hip joint showed internal rotation, opposite to the direction of movement. When considering changes of direction from the viewpoint of rotary motion of segments on horizontal planes, reverse torque is necessary to cancel out the torque in the direction of movement. In other words, regarding the change of direction examined in this study, the hip joint created reverse torque against the torque in the direction of movement created by the external rotation of the knee joint. Furthermore, as for the trunk, the possibility was suggested that it adjusts movements by creating torque in the opposite direction to the hip joints by rotating to the left.

To date, when discussing direction change, the function of the hip joint and leg have attracted attention ${ }^{14)}$. However, through examining tandem gait on a sine wave walkway, knee joint rotation was also found to be important. In addition, the possibility of its use in balance evaluation was shown. Regarding each joint, when a certain movement is difficult to execute despite having a reference mobile range, a decline in function can be inferred from the patterns of movement of each joint. Nevertheless, we have not verified in this study whether knee joint rotation is an active movement or whether it is a passive movement. In future research, we plan to go forward with electromyographical analysis.

\section{REFERENCES}

1) Oida Y, Arao T, Nishijima Y, et al.: Method of measuring physical activity of daily living in elderly and reference values for evaluation by sex and age group. Bull Phys Fit Res Inst, 1994, 86: 31-36.

2) Masuda T, Watanabe R, Muto M, et al.: Examination of daily life activity test in elderly: measuring the time in $10 \mathrm{~m}$ zigzag walking. Phys Exer Sport Sci, 2004, 10: 69-74.

3) Masuda S, Suganuma K, Kaneko C, et al.: Prediction of falls using 3-m zigzag walk test. J Phys Ther Sci, 2013, 25: 1051-1054. [Medline] [CrossRef]

4) Abe T, Ogawa M, Loenneke JP, et al.: Relationship between site-specific loss of thigh muscle and gait performance in women: the HIREGASAKI study. Arch Gerontol Geriatr, 2012, 55: e21-e25. [Medline] [CrossRef]

5) Abe T, Ogawa M, Thiebaud RS, et al.: Is muscle strength ratio a criterion for diagnosis of site-specific muscle loss? Geriatr Gerontol Int, 2014, 14: 837-844. [Medline] [CrossRef]

6) Kawakami S, Suzuki H, Kikuchi A, et al.: Study of postural control during one-legged stance: relationship between COG and COP. J Phys Ther Sci, 2015, 30: 399-403.

7) Kawakami S, Suzuki H, Tanaka N, et al.: COP control during one-legged stance: influence of femoral leg rotation function. Ann Rep Tohoku Phy Ther, 2015, 27: $40-44$.

8) Ito M, Takahashi Y, Seto N: Movement of direction change when walking. J Kansai Phys Ther, 2015, 15: $23-27$.

9) Yamazaki W, Tanino Y: Lower extremity joint torque during a change in direction when walking. J Kansai Phys Ther, 2015, 15: 67-74.

10) Senju H: Activities of Daily Living (ADL), 2nd ed. Fukuoka: Kyushu Shinryo Bunko, 2011, pp 49-54.

11) Ross R: A quantitative study of rotation of the knee joint in man. Anat Rec, 1932, 52: 209-223. [CrossRef]

12) Ouellet R, Lévesque HP, Laurin CA: The ligamentous stability of the knee: an experimental investigation. Can Med Assoc J, 1969, 100: 45-50. [Medline]

13) Mossberg KA, Smith LK: Axial rotation of the knee in women. J Orthop Sports Phys Ther, 1983, 4: 236-240. [Medline] [CrossRef]

14) Wang Y, Srinivasan M: Stepping in the direction of the fall: the next foot placement can be predicted from current upper body state in steady-state walking Biol Lett, 2014, 10: 1-5. [Medline] [CrossRef] 